\title{
Cutting balloon to treat post-stenting intramural hematoma during ST elevation myocardial infarction
}

\author{
Clément Servoz, Jacques Monségu, Mohamed Abdellaoui, Benjamin Faurie \\ Cardiovascular Institute, Groupe Hospitalier Mutualiste, Grenoble, France
}

Adv Interv Cardiol 2021; 17, 1 (63): 114-115

DOI: https://doi.org/10.5114/aic.2021.104779

A 71-year-old woman was admitted to our institution for an ongoing inferior ST-elevation myocardial infarction (STEMI). TIMI 3 flow of a mid-right coronary artery (RCA) thrombotic occlusion was restituted with balloon angioplasty (Sapphire II NC $3 \times 15 \mathrm{~mm}$, OrbusNech) and stenting at $9 \mathrm{~atm}$ (Ultimaster Tansei $3.5 \times 28 \mathrm{~mm}$, TERUMO) (Figure $1 \mathrm{~A}$ ). Post-stenting angiogram revealed a distal stent edge stenosis which was not restituted after intracoronary nitroglycerin excluding vasospasm (Figure $1 \mathrm{~B}$ ). Moreover, this lesion was evolutive: the stenosis aspect and degree became worse. As the dissection mechanism was suspected, we treated this lesion with a second stent. While stenting, we observed a distal migration of the sub-intimal contrast dye that made us think about a probable iatrogenic hematoma extension. A tight distal RCA stenosis was revealed on angiogram with clear subintimal dye trapping and extraluminal compression while the patient had recurrence of chest pain and inferior ST-segment elevation (Figure $1 \mathrm{C}$ ). The diagnosis of iatrogenic subintimal hematoma defined as blood accumulation in the medial space without distinguishable entry or exit points was considered [1]. We thought that additional stenting could worsen the situation by moving forward the hematoma and jeopardize the RCA crux and its distal flow. Intracoronary imaging would have confirmed the diagnosis but was not performed in the context of emergency so as not to delay revascularization. Hence, we decided to fenestrate this hematoma with a cutting balloon (CB) angioplasty in order to evacuate blood from the subintimal space as previously described for spontaneous hematoma [2]. At the site of hematoma, three CB (Wolverine $3 \times 10 \mathrm{~mm}$, Boston Scientific) incremental inflations at 6,8 then $11 \mathrm{~atm}$ were required to obtain the awaited effect and recover a good flow without subintimal contrast trapping anymore. Chest pain and ST elevation disappeared immediately; nevertheless, we stented this fenestrated area (Ultimaster Tansei $3.5 \times 28 \mathrm{~mm}$,
TERUMO) (Figure $1 \mathrm{D}$ ). Ultra-sensitive troponin maximum level was $237594 \mathrm{ng} / \mathrm{l}$ and the patient was discharged from hospital after 5 days with a limited inferior wall akinesia on echocardiography. Frequently, after stent implantation when stenosis appears at the stent edge, diagnosis and management of such an issue is difficult. In some exceptional situations when true lumen compression leads to ischemia, cutting balloon angioplasty at the site induces a fenestration between the true lumen and hematoma. This situation has been described in cases of spontaneous coronary artery dissection or during chronic total occlusion revascularization $[3,4]$. In the context of ventricular arrhythmias or clinical instability as in our case, diagnosis should be confirmed by intracoronary imaging: intravascular ultrasound (IVUS) or optical coherence tomography (OCT), even though OCT risk is a distal propagation of the hematoma. To fenestrate the hematoma while minimizing the risk of distality extension, a size ratio of 1 : 1 cutting balloon to artery diameter ratio at nominal pressure should be recommended. Nevertheless, CB inflation pressure could be increased to rated burst pressure until obtaining the awaited effect as in our example. We could also advocate increasing CB size in case of failure to obtain fenestration. Some case reports have described successful angioplasty without stenting. In our case, the high thrombotic burden and close location to the RCA bifurcation led us to stent this wounded area. Coronary intra-mural hematoma during STEMI percutaneous intervention revascularization is a rare complication. Here we report a case to illustrate an indication for cutting balloon on post-stenting intramural hematoma during STEMI.

\section{Conflict of interest}

Benjamin Faurie is consultant for Boston Scientific, Jacques Monségu is consultant for Terumo. None for this case for all other authors. 

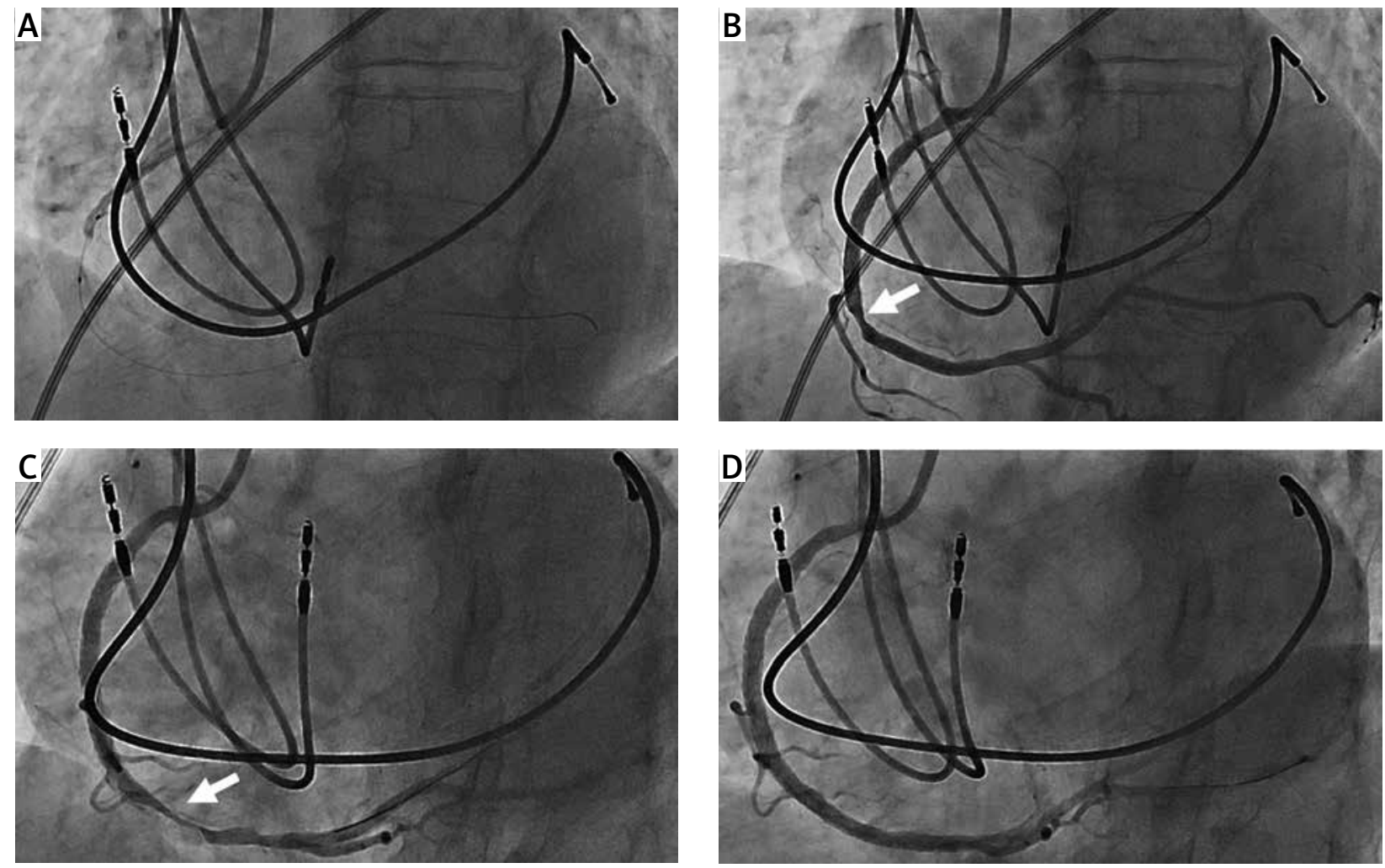

Figure 1. A - Coronary angiogram showing mid-RCA occlusion just before balloon angioplasty. B - Persistent significant stenosis at the stent edge did not resolve with intracoronary nitroglycerin. C - Post-stenting angiogram exhibiting propagation of compressive intramural hematoma. D - Final result showing successful angioplasty

\section{References}

1. Maehara A, Mintz GS, Bui AB, et al. Incidence, morphology, angiographic findings, and outcomes of intramural hematomas after percutaneous coronary interventions: an intravascular ultrasound study. Circulation 2002; 105: 2037-42.

2. McGrath BM, Vo MN. Novel use of cutting balloon to manage compressive subintimal hematoma during left main stenting in a patient with spontaneous coronary artery dissection. Clin Case Rep 2018; 6: 1291-5.

3. Alkhouli M, Cole M, Ling FS. Coronary artery fenestration prior to stenting in spontaneous coronary artery dissection. Catheter Cardiovasc Interv 2016; 88: E23-7.

4. Vo MN, Brilakis ES, Grantham JA. Novel use of cutting balloon to treat subintimal hematomas during chronic total occlusion interventions. Catheter Cardiovasc Interv 2018; 91: 53-6. 